\title{
Dyspnea perception in cystic fibrosis patients
}

\author{
B. Ziegler ${ }^{1}$, A.K. Fernandes ${ }^{2}$, P.R.S. Sanches ${ }^{3}$, D.P. Silva Junior ${ }^{3}$, \\ P.R.O. Thomé ${ }^{3}$ and P.T.R. Dalcin ${ }^{4}$ \\ ${ }^{1}$ Fisioterapia, Universidade Federal do Rio Grande do Sul, and Serviço de Pneumologia, Hospital de Clínicas de Porto Alegre, \\ Universidade Federal do Rio Grande do Sul, Porto Alegre, RS, Brasil \\ ${ }^{2}$ Emergência, Universidade Federal do Rio Grande do Sul, and Serviço de Pneumologia, Hospital de Clínicas de Porto Alegre, \\ Universidade Federal do Rio Grande do Sul, Porto Alegre, RS, Brasil \\ ${ }^{3}$ Engenharia Biomédica, Hospital de Clínicas de Porto Alegre, Porto Alegre, RS, Brasil \\ ${ }^{4}$ Faculdade de Medicina, Universidade Federal do Rio Grande do Sul, and Serviço de Pneumologia, \\ Hospital de Clínicas de Porto Alegre, Porto Alegre, RS, Brasil
}

\begin{abstract}
We evaluated dyspnea perception in cystic fibrosis patients compared with normal subjects, during an inspiratory resistive loading test and 6-min walk test. We also evaluated the correlation between dyspnea scores induced by resistive loads and by the 6-min walk test. In this prospective, cross-sectional study, 31 patients with cystic fibrosis ( $\geqslant 15$ years of age) and 31 age-, gender-, and ethnicity-matched healthy volunteers (20 females and 11 males per group) underwent inspiratory resistive loading, spirometry, and the 6-min walk test. As the magnitude of the inspiratory loads increased, dyspnea scores increased $(P<0.001)$, but there was no difference between groups in dyspnea score $(P=0.654)$. Twenty-six $(84 \%)$ normal subjects completed all the resistive loads, compared with only $12(39 \%)$ cystic fibrosis patients $(\mathrm{P}<0.001)$. Dyspnea scores were higher after the 6-min walk test than at rest $(P<0.001)$, but did not differ between groups $(P=0.080)$. Post-6-min walk test dyspnea scores correlated significantly with dyspnea scores induced by resistive loads. We conclude that dyspnea perception induced in cystic fibrosis patients by inspiratory resistive loading and by 6-min walk test did not differ from that induced in normal subjects. However, cystic fibrosis patients discontinued inspiratory resistive loading more frequently. There were significant correlations between dyspnea perception scores induced by inspiratory resistance loading and by the 6-min walk test. This study should alert clinicians to the fact that some cystic fibrosis patients fail to discriminate dyspnea perception and could be at risk for delay in seeking medical care.
\end{abstract}

Key words: Cystic fibrosis; Dyspnea perception; Pulmonary function test; Six-min walk test; Inspiratory resistive load testing

\section{Introduction}

Dyspnea is the subjective experience of breathing discomfort that consists of qualitatively distinct sensations that vary in intensity. This symptom has multidimensional aspects involving physiological, psychological, social, and environmental factors that result in a behavioral response (1). Assessment of multidimensional aspects of dyspnea has become more important in recent years. Decreased perception of dyspnea in patients with asthma has been associated with an increase in morbidity and mortality (2).

For individuals with cystic fibrosis (CF), pulmonary disease is associated with increased morbidity, mortality, and poor prognosis (3). Dyspnea is a frequent symptom in CF patients with pulmonary impairment, and is often accompanied by physical inactivity, decreased exercise capacity, and quality of life (4-7).

Few studies have evaluated dyspnea perception during exercise tests in CF patients $(3,8-11)$. Chetta et al. (9) showed a relationship between dyspnea perception and peripheral oxygen saturation during the 6-min walk test (6MWT) in CF adults with mild and moderate lung disease. Moorcroft et al. (11) reported a relationship between breathlessness and maximal exercise capacity, but only in CF patients with severe lung disease.

Inspiratory resistive loading is frequently used in dyspnea research to increase the effort and work of breathing (12-18). Despite extensive use of resistive loads in physiological research, and the frequent discrepancy between a respiratory patient's self-reported dyspnea and the extent of pathophysiology $(14,15,17)$, no study has investigated the effects of respiratory CF disease on scores of perceived dyspnea.

In previous research (19), we found that subjective perception of the disease severity in CF patients correlated only moderately with objective measures of

Correspondence: B. Ziegler, Rua Miranda e Castro, 70/204, 90040-280 Porto Alegre, RS, Brasil. E-mail: brunaziegler@yahoo.com.br 
disease severity, such as clinical score and pulmonary function tests. In another study (20), we observed that CF patients reported only mild to moderate dyspnea during the 6MWT, despite significantly impaired pulmonary function. One hypothesis generated from these findings was that the subjective perception of the patients discriminates the severity of their disease when it is mild. As the disease progresses, from moderate to severe, CF patients cease to discriminate progressive health impairments, especially dyspnea perception. Therefore, in this study we tested the hypothesis that CF patients would show a blunted perception of dyspnea when compared to normal subjects.

The main objective of this study was to compare the dyspnea perception scores in CF patients with those of normal subjects during ventilation in an inspiratory resistive load system and during the 6MWT. A secondary objective was to assess the correlation between Borg dyspnea scores during the 6MWT and during inspiratory resistive loading

\section{Material and Methods}

\section{Study design}

This cross-sectional study evaluated dyspnea perception in data collected prospectively from CF patients and normal subjects. We performed inspiratory resistive loading and evaluated the perceived dyspnea scores, maximal respiratory pressures, pulmonary function tests, nutritional evaluation, and 6MWT during the same day in each patient and normal subject. The study was approved by the Hospital de Clínicas de Porto Alegre (HCPA) Ethics Committee (08-063), and written informed consent was obtained from each volunteer or legally responsible person in the case of subjects younger than 18 years or age.

\section{Population}

Patients were recruited from the Adult CF Program at HCPA. A total of 35 CF patients were screened between January and November 2009. Four CF patients refused to participate. The $31 \mathrm{CF}$ patients who volunteered were sequentially enrolled during the study period. The diagnosis was confirmed according to criteria established by the Cystic Fibrosis Foundation Consensus Panel (21). All patients were clinically stable, defined as no recent change (in the last 30 days) in medication, with at least 30 days since completion of last intravenous or oral antibiotics for pulmonary exacerbation. Patients were excluded if they were pregnant, had a cardiac or orthopedic disease or a traumatic injury, or could not perform the diagnostic tests because of their clinical condition. A total of 36 normal subjects, recruited with notices posted in the hospital and by electronic announcements, were screened between February 2010 and December 2010; two were excluded because of abnormal spirometry values and three were excluded because they failed to complete all required examinations. The 31 normal subjects were paired with CF patients by gender, age, and ethnicity. Pregnancy, acute respiratory tract complaints, current or past smoking, or any chronic medical conditions such as asthma, chronic pain, cardiac or orthopedic disease, or traumatic injury were exclusion criteria. There were 20 females and 11 males per group. The mean age of the patients and normal subjects was $25.7 \pm 8.6$ and $26.1 \pm 8.8$ years, respectively, and ranged from 15 to 53 years. All were Caucasians.

\section{Measurements and procedures}

Normal subjects and CF patients were given a dyspnea perception test using an inspiratory resistive load system (22). Before the test, participants were familiarized with the apparatus and measurement procedures. After standardized instructions, volunteers were seated in a comfortable chair and acclimatized to the setting. Wearing a nose clip, volunteers breathed through a mouthpiece in a system comprised of a two-way nonrebreathing valve (Hans-Rudolph, USA). A plastic circular mouthpiece, with eight different orifices generated inspiratory loads of increasing magnitude (@0.6, 7.0, 15.0, 25.0, $46.7,67.0$, and $78.0 \mathrm{cmH} \mathrm{CH}_{2} \mathrm{O} \cdot \mathrm{L}^{-1} \cdot \mathrm{s}^{-1}$, calculated according to a constant flow of $300 \mathrm{~mL} / \mathrm{s}$ ). The sensation of dyspnea was assessed during ventilation with increasing inspiratory resistive loads. After breathing at each level of resistance for $2 \mathrm{~min}$, the subjects were questioned about their feeling of shortness of breath (dyspnea) using the modified Borg scale (23), ranging from 0 (no dyspnea) to 10 (maximal dyspnea). To monitor the effects of dyspnea induction, inspiratory pressure, inspiratory time, and respiratory frequency were measured continuously at the mouthpiece using computer software developed by the engineering service of HCPA. Expiration was not loaded. Subjects were free to choose their breathing rate, volume, and flow to have as natural a breathing pattern as possible.

The functional capacity of subjects was measured by performing the 6MWT according to the guidelines of the American Thoracic Society (24). The distance the patient was able to walk in 6 min was determined in a 30-m long corridor, following a standardized protocol. The patients were instructed to walk as far as possible for 6 min under the supervision of a physiotherapist. The physiotherapist encouraged subjects with the standardized statements: "you are doing well" or "keep up the good work" but were asked not to use other phrases. The total distance walked was recorded and the initial and the final oxygen saturation $\left(\mathrm{SpO}_{2}\right)$ were measured by pulse oximetry (NPB-40; Nellcor Puritan Bennett, USA). Dyspnea scores were measured pre- and post-6MWT using the modified Borg scale (23).

Pulmonary function tests were carried out with a computerized spirometer (MasterScreen, v 4.31, Jaeger, Germany). The forced vital capacity (FVC), forced 
expiratory volume in $1 \mathrm{~s}\left(\mathrm{FEV}_{1}\right)$, and $F E V_{1} / F V C$ were measured three times, and the best trial was recorded. All parameters are reported as percentages of predicted values for age, stature, and gender (25). Nutritional status was assessed as body mass index (BMI). BMI was calculated by dividing the subject's body mass by the square of their height in meters $\left(\mathrm{kg} / \mathrm{m}^{2}\right)$.

Maximal respiratory pressures were used as indices of respiratory muscle strength. Pressure measurements were made in the seated position by the digital manometer model MVD $-300 /+300$, version 1.0 (Microhard, Brazil). All subjects wore nose clips and pressed their lips tightly against the mouthpiece during the pressure measurements to prevent air leakage.

The maximal inspiratory pressure (MIP) was measured at residual volume and the maximal expiratory pressure (MEP) was measured at total lung capacity. The pressures measured were maintained for at least $1 \mathrm{~s}$. Five repeated determinations were made with a suitable rest pause until a plateau value was reached and no further learning effect was seen. Once the operator was satisfied, the maximum values of two maneuvers that varied by less than $10 \%$ were recorded. The MIP and MEP are reported in $\mathrm{CmH}_{2} \mathrm{O}$ and as percentage of the predicted value. We obtained our predicted values for adolescents from Wilson et al. (26) and for adults from Neder et al. (27).

\section{Statistical analysis}

Data are reported as number and proportion of cases, means $\pm S D$, or median (interquartile range). Categorical comparisons were performed by a chi-square test with adjusted standardized residuals. Continuous variables with a normal distribution were compared with the paired samples $t$-test. Ordinal variables or continuous variables without a normal distribution were compared with the Wilcoxon signed-rank test. Generalized linear analysis with estimating equations was used for comparison of repeated measurements between CF patients and normal subjects during inspiratory resistive load testing. KaplanMeier curves, stratified by group, either CF patients or normal subjects, were compared with the log rank test to compare the completion of the dyspnea perception test during inspiratory resistive loads. General linear models for repeated measures were used to compare the dyspnea scores between groups before and after the 6MWT. Correlations were determined using Spearman's rank correlation coefficient. Data analysis was performed with the SPSS software, version 18.0. The statistical significance level was set at $\mathrm{P}<0.05$. All probabilities reported were two-tailed.

We calculated the sample size based on the study of Kikuchi et al. (2) using the inspiratory resistance of $20.0 \mathrm{cmH}_{2} \mathrm{O} \cdot \mathrm{L}^{-1} \cdot \mathrm{s}^{-1}$. To detect an effect of two points in the Borg dyspnea score, with a standard deviation of two points, $\alpha=0.05$ and $1-\beta=90 \%$, an adequate sample size was found to be at least 23 subjects per group.

\section{Results}

There were significant differences between CF patients and normal subjects in $\mathrm{BMI}, \mathrm{FEV}_{1} \%$ predicted, FVC\% predicted, $\mathrm{FEV}_{1} / \mathrm{FVC} \%$ predicted, distance walked in the 6MWT, and oxygen desaturation during 6MWT. There were no significant differences in age, gender, education level, MIP, MEP, post-test Borg dyspnea score, and post-test Borg leg fatigue score (Table 1).

Figure 1 shows the group-specific (CF patients vs normal subjects) generalized linear analysis with estimating equations by inspiratory resistive loads for the Borg dyspnea score, inspiratory pressure, respiratory frequency, and $\mathrm{SpO}_{2}$.

Figure 2 presents the Kaplan-Meier analysis of interruption of the dyspnea perception test with increasing inspiratory resistive loads, in both CF patients and normal subjects. There was a significant difference between the Kaplan-Meier curves of the two groups. Twenty-six (84\%) normal subjects performed the whole test compared to only $12(39 \%)$ CF patients.

Five normal subjects discontinued before completing all the inspiratory resistive loads for the following symptoms: dyspnea $(n=1)$, respiratory fatigue $(n=1)$, chest tightness $(n=1)$, headache $(n=1)$, drooling $(n=1)$, and dry throat $(n=1)$. Nineteen CF patients discontinued the dyspnea perception inspiratory resistive loading test for the following reasons: dyspnea $(n=12)$, respiratory fatigue $(n=8)$, dry throat $(n=3)$, cough $(n=3)$, psychological symptoms $(n=2)$, drooling $(n=2)$, headache $(n=1)$, and pain in the accessory respiratory muscles $(n=1)$. In CF patients, there were no significant differences in $\mathrm{FEV}_{1} \%(\mathrm{P}=0.555)$ and at-rest $\mathrm{SpO}_{2}(\mathrm{P}=0.160)$ between those who discontinued the test and those who performed the whole test.

Figure 3 shows group-specific (CF patients vs normal subjects) general linear models for repeated measures during the 6MWT for Borg dyspnea score, Borg fatigue score, $\mathrm{SpO}_{2}$, and respiratory frequency.

In normal subjects, post-6MWT Borg dyspnea scores were significantly correlated with Borg dyspnea scores induced by the higher inspiratory loads. In CF patients, post-6MWT Borg dyspnea scores were significantly correlated with Borg dyspnea scores induced by lower resistances (Supplementary Table S1).

\section{Discussion}

The main finding of this cross-sectional study was that CF patients reported dyspnea perception scores similar to those of normal subjects in response to inspiratory resistive loads and 6MWT, even though these CF patients had moderate to severe impairment of lung function, lower at-rest $\mathrm{SpO}_{2}$, and significant desaturation during exercise. Compared to normal subjects, the CF patients discontinued inspiratory resistive loading more frequently and 
Table 1. Characteristics of cystic fibrosis (CF) patients and normal subjects.

\begin{tabular}{|c|c|c|c|}
\hline Variable & $C F(n=31)$ & Normal $(n=31)$ & $\mathrm{P}$ \\
\hline Age (years) & $25.7 \pm 8.6$ & $26.1 \pm 8.8$ & \\
\hline Gender (male/female) & $11 / 20$ & $11 / 20$ & \\
\hline BMI $\left(\mathrm{kg} / \mathrm{m}^{2}\right)$ & $20.7 \pm 2.4$ & $22.9 \pm 3.3$ & 0.004 \\
\hline \multicolumn{4}{|l|}{ Educational level [n (\%)] } \\
\hline$<8$ years of school & $2(6.5)$ & $2(6.5)$ & \\
\hline $8-13$ years of school & $25(80.6)$ & $19(61.3)$ & \\
\hline$\geqslant 13$ years of school & $4(12.9)$ & $10(32.2)$ & \\
\hline MIP (\%predicted) & $83.7 \pm 21.5$ & $88.5 \pm 26.4$ & \\
\hline MEP (\%predicted) & $97.1 \pm 22.2$ & $97.1 \pm 24.6$ & \\
\hline $\mathrm{FEV}_{1}$ (\%predicted) & $53.4 \pm 21.1$ & $93.5 \pm 11.7$ & $<0.001$ \\
\hline FVC (\%predicted) & $66.7 \pm 16.4$ & $94.6 \pm 11.7$ & $<0.001$ \\
\hline FEV $1 / F V C$ (\%predicted) & $79.3 \pm 17.7$ & $99.2 \pm 8.5$ & $<0.001$ \\
\hline \multicolumn{4}{|l|}{ 6MWT } \\
\hline Walked distance (m) & $517.0 \pm 100.0$ & $577.5 \pm 76.1$ & 0.009 \\
\hline At-rest $\mathrm{SpO}_{2}(\%)$ & $96.0 \pm 2.1$ & $98.2 \pm 0.9$ & $<0.001$ \\
\hline Post-test $\mathrm{SpO}_{2}(\%)$ & $93.0 \pm 5.9$ & $97.8 \pm 1.6$ & $<0.001$ \\
\hline Oxygen desaturation (\%) & $-3.0 \pm 4.8$ & $-0.4 \pm 1.4$ & 0.005 \\
\hline At-rest Borg dyspnea score & $0.3(0-4)$ & $0.1(0-1)$ & \\
\hline Post-test Borg dyspnea score & $1.9(0-9)$ & $1.1(0-4)$ & \\
\hline At-rest Borg leg fatigue score & $0.3(0-2)$ & $0.1(0-2)$ & \\
\hline Post-test Borg leg fatigue score & $1.8(0-7)$ & $1.9(0-7)$ & \\
\hline
\end{tabular}

Data are reported as mean \pm SD or median (interquartile range) unless otherwise indicated. $\mathrm{n}$ : number of cases; BMI: body mass index; $\mathrm{FEV}_{1}$ : forced expiratory volume in the first second; FVC: forced vital capacity; 6MWT: 6-min walk test; MIP: maximal inspiratory pressure; MEP: maximal expiratory pressure; $\mathrm{SpO}_{2}$ : peripheral oxygen saturation. Only significant $\mathrm{P}$ values $(\mathrm{P} \leq 0.05)$ are reported (paired-samples $t$-test for continuous variables, Wilcoxon signed-rank test for ordinal variables or chi-square test for categorical variables).

walked shorter distances in the 6MWT; these findings may reflect another dimension of functional limitation in CF patients. During resistive loading, CF patients and normal subjects generated similar inspiratory pressures and also ventilated with similar respiratory frequency. Secondarily, we observed moderate to high correlations between dyspnea perception scores induced by inspiratory resistance loads and those induced by the 6MWT. The greatest correlations were between Borg dyspnea scores post-6MWT and those induced by inspiratory resistive loads of 15.0, 25.0, 46.7, 67.0 and $78.0 \mathrm{cmH}_{2} \mathrm{O} \cdot \mathrm{L}^{-1} \cdot \mathrm{s}^{-1}$. In CF patients, Borg dyspnea scores post-6MWT correlated most strongly with those induced by the lower inspiratory resistive loads; in contrast, correlations in normal subjects were greatest with higher loads.

A previous study (6) reported that chronic dyspnea in CF correlated poorly with standard measures of pulmonary function. We also observed that CF patients reported only mild to moderate dyspnea during the 6MWT, despite significant impairment in pulmonary function, suggesting that these patients had a blunted perception of dyspnea (20). In CF patients, dyspnea is of particular interest, since it may be a sign of severe pulmonary disease or nonpulmonary disease. Those patients with poor perception of dyspnea could be at risk of higher morbidity and mortality due to delays in seeking medical care.
In this study, dyspnea was successfully induced by breathing through inspiratory resistive loads of increasing magnitude, which significantly increased inspiratory pressure in both CF patients and normal subjects. These findings correspond to the reported typical effects of resistive loads, which increase the work and effort of breathing (18). We used a protocol with seven different inspiratory loads, ranging from 0.6 to $78.0 \mathrm{cmH}_{2} \mathrm{O} \cdot \mathrm{L}^{-1} \cdot \mathrm{s}^{-1}$. After breathing at each level of resistance for $2 \mathrm{~min}$ without resting, volunteers were asked to express their feeling of dyspnea. The fact that we performed the test without pausing may explain why the test was discontinued so frequently. Although CF patients reported scores of dyspnea perception similar to those of normal subjects, they discontinued the test more frequently than did normal subjects (61 vs 16\%,), showing that increasing inspiratory loads and/or dyspnea had a greater functional impact in the CF group.

It is worth noting that our approach differed from previous studies $(16,17,22,28)$ in that we did not apply a randomized sequence of inspiratory resistive loads. In this study, resistive loads of progressive magnitude were presented to mirror the character of naturally occurring dyspnea. However, randomized presentations of different loads might be an alternative method that avoids the perception by patients of the progressive magnitude of the loads. 

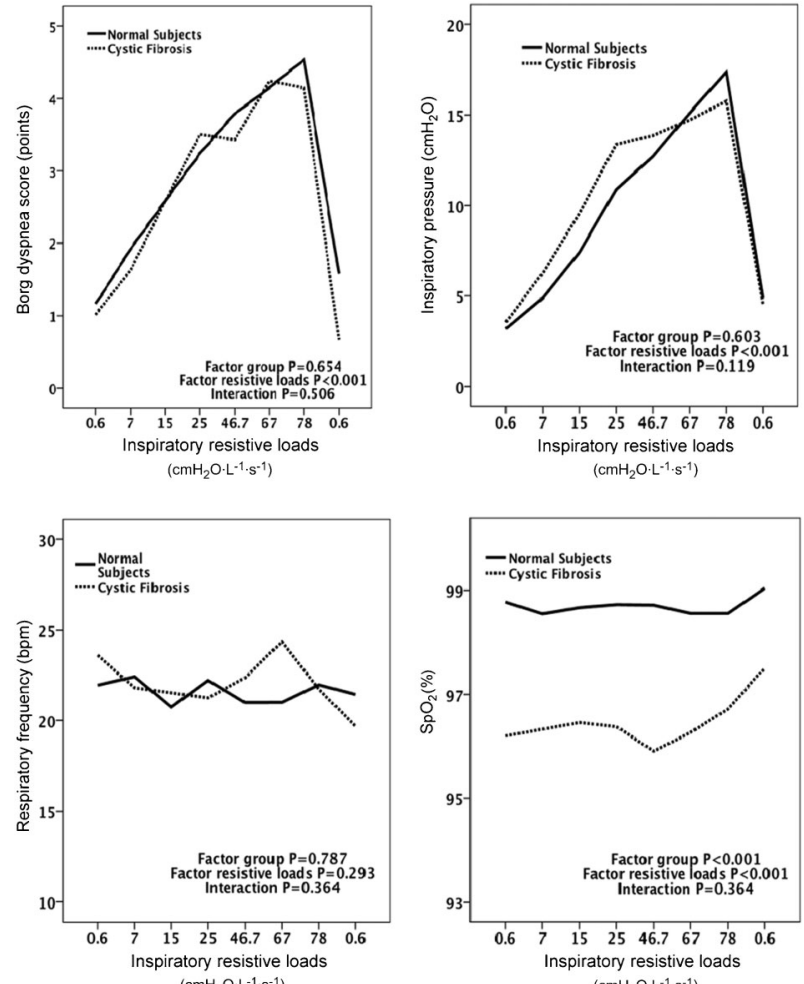

Figure 1. Group-specific (cystic fibrosis patients vs normal subjects) generalized linear analysis with estimating equations by inspiratory resistive loads for Borg dyspnea score, inspiratory pressure, respiratory frequency, and peripheral oxygen saturation $\left(\mathrm{SpO}_{2} \%\right)$.

When comparing CF patients with normal subjects during the 6MWT, we observed similar Borg dyspnea scores and Borg fatigue scores, even though CF patients had lower $\mathrm{SpO}_{2}$, higher respiratory frequency, and walked shorter distances during the test. Chetta et al. (9) assessed exercise capacity by 6MWT in a group of 25 adult CF patients with mild and moderate lung disease and in 22 healthy volunteers. In contrast to our research, CF patients walked similar distances but presented more breathlessness compared to the control group. CF patients also experienced a significant decrease in oxygen saturation during the 6MWT. Moorcroft et al. (11) performed cycloergometry in 104 CF patients and 27 controls. Even when performing a maximal exercise test, they did not find differences between their groups for breathlessness or muscle effort. However, when they classified CF by the severity of lung disease, they found higher breathlessness and lower muscle effort in the group with $\mathrm{FEV}_{1}<40 \%$.

Another interesting finding were the positive correlations between post-6MWT Borg dyspnea scores and Borg dyspnea scores induced by inspiratory resistive loads. The correlation between two different dyspnea responses, after

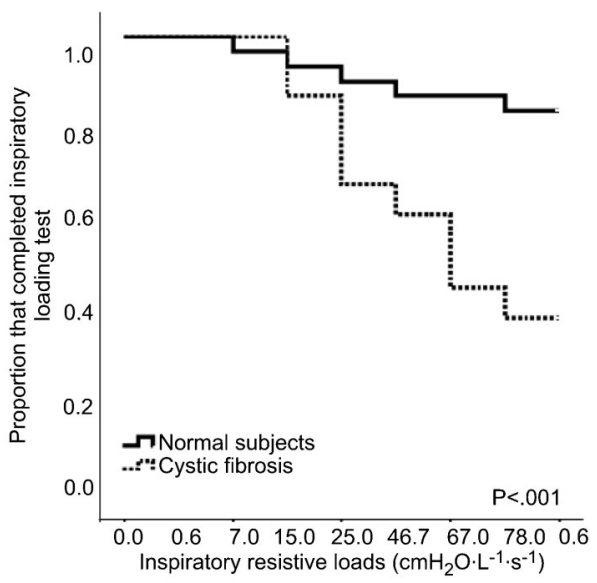

Figure 2. Kaplan-Meier analysis for the interruption of the dyspnea perception test by increasing inspiratory resistive loads stratified for cystic fibrosis patients and normal subjects.

exercise testing and during static inspiratory resistive loads, demonstrated the consistency of the magnitude of dyspnea perception and the importance of regular assessment of dyspnea perception in patients with chronic lung disease.

Dyspnea perception in patients with pulmonary disease could be influenced by intrinsic factors such as hypoxemia, bronchoconstriction, inspiratory muscle strength, limb muscle fatigue, and hyperinflation $(1,12,13,29)$. CF patients could be influenced by these factors, especially patients with severe lung disease and hyperinflation; they
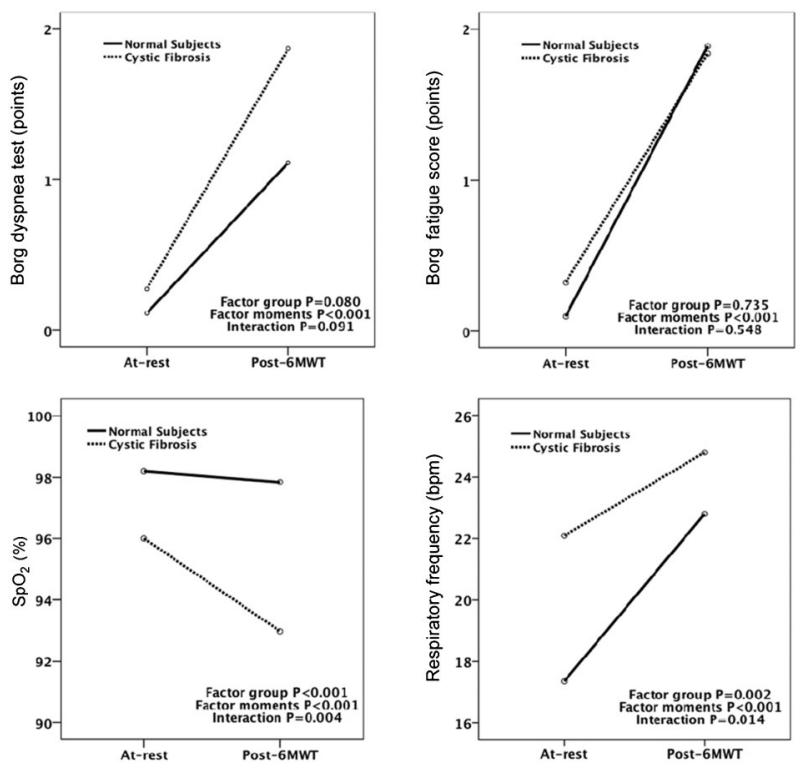

Figure 3. Group-specific (cystic fibrosis patients vs normal subjects) general linear model for repeated measures during the 6 -min walk test (6MWT) for Borg dyspnea score, Borg fatigue score, peripheral oxygen saturation $\left(\mathrm{SpO}_{2} \%\right)$, and respiratory frequency. 
could develop an adaptive mechanism affecting inspiratory muscle strength. Huang et al. (13) performed inspiratory strength training in 12 healthy volunteers, with a mean age of 26 years, and evaluated dyspnea perception with a similar apparatus. These researchers concluded that increased respiratory muscle strength could increase the ability of pulmonary patients to compensate for increased respiratory loads and modulate the threshold for detection of changes in pulmonary mechanics.

The present study has some limitations. First, the cross-sectional study design did not allow the examination of temporal relationships between dyspnea perception and lung function decline. Second, our sample was small, so future investigations in larger cohorts are needed to confirm our findings.

The clinical implication of this study was that some CF patients fail to discriminate progressive health impairments, especially dyspnea perception. When evaluating clinical severity, clinicians should take this finding into account. Patients with a poor perception of dyspnea could

\section{References}

1. Parshall MB, Schwartzstein RM, Adams L, Banzett RB, Manning HL, Bourbeau J, et al. An official American Thoracic Society statement: update on the mechanisms, assessment, and management of dyspnea. Am J Respir Crit Care Med 2012; 185: 435-452, doi: 10.1164/rccm.201111-2042ST.

2. Kikuchi Y, Okabe S, Tamura G, Hida W, Homma M, Shirato K, et al. Chemosensitivity and perception of dyspnea in patients with a history of near-fatal asthma. N Engl J Med 1994; 330: 1329-1334, doi: 10.1056/NEJM199405123301901.

3. Mogayzel PJ Jr, Naureckas ET, Robinson KA, Mueller G, Hadjiliadis D, Hoag JB, et al. Cystic fibrosis pulmonary guidelines. Chronic medications for maintenance of lung health. Am J Respir Crit Care Med 2013; 187: 680-689, doi: 10.1164/rccm.201207-11600E.

4. Sawicki GS, Sellers DE, Robinson WM. Self-reported physical and psychological symptom burden in adults with cystic fibrosis. J Pain Symptom Manage 2008; 35: 372-380, doi: 10.1016/j.jpainsymman.2007.06.005.

5. Stenekes SJ, Hughes A, Gregoire MC, Frager G, Robinson WM, McGrath PJ. Frequency and self-management of pain, dyspnea, and cough in cystic fibrosis. J Pain Symptom Manage 2009; 38: 837-848, doi: 10.1016/j.jpainsymman.2009.04.029.

6. de Jong W, van der Schans CP, Mannes GP, van Aalderen WM, Grevink RG, Koeter GH. Relationship between dyspnoea, pulmonary function and exercise capacity in patients with cystic fibrosis. Respir Med 1997; 91: 41-46, doi: 10.1016/S0954-6111(97)90135-9.

7. Stevens D, Stephenson A, Faughnan ME, Leek E, Tullis E. Prognostic relevance of dynamic hyperinflation during cardiopulmonary exercise testing in adult patients with cystic fibrosis. J Cyst Fibros 2013 [Epub ahead of print]. doi: 10.1016/j.jcf.2013.04.010.

8. Leroy S, Perez T, Neviere R, Aguilaniu B, Wallaert B. Determinants of dyspnea and alveolar hypoventilation during exercise in cystic fibrosis: impact of inspiratory be at risk for delay in seeking medical care.

The results suggest that dyspnea perception induced in CF patients by inspiratory resistive loads and the 6MWT did not differ from that induced in normal subjects. However, compared to normal subjects, CF patients discontinued the inspiratory resistive loading more frequently, demonstrated greater oxygen desaturation, and walked shorter distances in the 6MWT. In addition, there was moderate to high correlation between the dyspnea perception scores induced by inspiratory resistance loading and by the 6MWT.

\section{Supplementary Material}

Click here to view [pdf]

\section{Acknowledgments}

We thank Vânia Naomi Hirakata for statistical analyses and Biomed Proofreading for English editing.

muscle endurance. J Cyst Fibros 2011; 10: 159-165, doi: 10.1016/j.jcf.2010.12.006.

9. Chetta A, Pisi G, Zanini A, Foresi A, Grzincich GL, Aiello M, et al. Six-minute walking test in cystic fibrosis adults with mild to moderate lung disease: comparison to healthy subjects. Respir Med 2001; 95: 986-991, doi: 10.1053/rmed.2001.1194.

10. Coelho CC, Aquino ES, de Almeida DC, Oliveira GC, Pinto $\mathrm{RC}$, Rezende IM, et al. Comparative analysis and reproducibility of the modified shuttle walk test in normal children and in children with cystic fibrosis. J Bras Pneumol 2007; 33: 168-174, doi: 10.1590/S1806-37132007000200011.

11. Moorcroft AJ, Dodd ME, Morris J, Webb AK. Symptoms, lactate and exercise limitation at peak cycle ergometry in adults with cystic fibrosis. Eur Respir J 2005; 25: 10501056, doi: 10.1183/09031936.05.00011404.

12. Grippo A, Carrai R, Chiti L, Bruni GI, Scano G, Duranti R. Effect of limb muscle fatigue on perception of respiratory effort in healthy subjects. J Appl Physiol 2010; 109: 367376, doi: 10.1152/japplphysiol.00608.2009.

13. Huang $\mathrm{CH}$, Martin $\mathrm{AD}$, Davenport PW. Effects of inspiratory strength training on the detection of inspiratory loads. Appl Psychophysiol Biofeedback 2009; 34: 17-26, doi: 10.1007/ s10484-008-9073-y.

14. von Leupoldt A, Mertz C, Kegat S, Burmester S, Dahme B. The impact of emotions on the sensory and affective dimension of perceived dyspnea. Psychophysiology 2006; 43: 382-386, doi: 10.1111/j.1469-8986.2006.00415.x.

15. von Leupoldt A, Ambruzsova R, Nordmeyer S, Jeske N, Dahme B. Sensory and affective aspects of dyspnea contribute differentially to the Borg scale's measurement of dyspnea. Respiration 2006; 73: 762-768.

16. Killian KJ, Bucens DD, Campbell EJ. Effect of breathing patterns on the perceived magnitude of added loads to breathing. J Appl Physiol 1982; 52: 578-584.

17. Knafelc M, Davenport PW. Relationship between magnitude 
estimation of resistive loads, inspiratory pressures, and the RREP P(1) peak. J Appl Physiol 1999; 87: 516-522.

18. von Leupoldt A, Dahme B. Differentiation between the sensory and affective dimension of dyspnea during resistive load breathing in normal subjects. Chest 2005; 128: 33453349, doi: 10.1378/chest.128.5.3345.

19. Dalcin PT, Rampon G, Pasin LR, Becker SC, Ramon GM, Oliveira VZ. Perception of disease severity in adult patients with cystic fibrosis. J Bras Pneumol 2009; 35: 27-34, doi: 10.1590/S1806-37132009000100005.

20. Ziegler B, Rovedder PM, Oliveira CL, Schuh SJ, Silva FA, Dalcin PT. Predictors of oxygen desaturation during the sixminute walk test in patients with cystic fibrosis. J Bras Pneumol 2009; 35: 957-965.

21. Rosenstein BJ, Cutting GR. The diagnosis of cystic fibrosis: a consensus statement. Cystic Fibrosis Foundation Consensus Panel. J Pediatr 1998; 132: 589-595, doi: 10.1016/S0022-3476(98)70344-0.

22. Orr RS, Jordan AS, Catcheside P, Saunders NA, McEvoy $\mathrm{RD}$. Sustained isocapnic hypoxia suppresses the perception of the magnitude of inspiratory resistive loads. J Appl Physiol 2000; 89: 47-55.

23. Borg GA. Psychophysical bases of perceived exertion. Med Sci Sports Exerc 1982; 14: 377-381.
24. ATS statement: guidelines for the six-minute walk test. $A m \mathrm{~J}$ Respir Crit Care Med 2002; 166: 111-117, doi: 10.1164/ ajrccm.166.1.at1102.

25. Miller MR, Hankinson J, Brusasco V, Burgos F, Casaburi R, Coates A, et al. Standardisation of spirometry. Eur Respir $J$ 2005; 26: 319-338, doi: 10.1183/09031936.05.00034805.

26. Wilson SH, Cooke NT, Edwards RH, Spiro SG. Predicted normal values for maximal respiratory pressures in Caucasian adults and children. Thorax 1984; 39: 535-538, doi: 10.1136/thx.39.7.535.

27. Neder JA, Andreoni S, Lerario MC, Nery LE. Reference values for lung function tests. II. Maximal respiratory pressures and voluntary ventilation. Braz $J$ Med Biol Res 1999; 32: 719-727.

28. Eckert DJ, Catcheside PG, Stadler DL, McDonald R, Hlavac MC, McEvoy RD. Acute sustained hypoxia suppresses the cough reflex in healthy subjects. Am J Respir Crit Care Med 2006; 173: 506-511, doi: 10.1164/rccm. 200509-1455OC.

29. Monteiro MB, Berton DC, Moreira MA, Menna-Barreto SS, Teixeira PJ. Effects of expiratory positive airway pressure on dynamic hyperinflation during exercise in patients with COPD. Respir Care 2012; 57: 1405-1412, doi: 10.4187/ respcare.01481. 